\title{
RATIONAL FIRST INTEGRALS FOR POLYNOMIAL VECTOR FIELDS ON ALGEBRAIC HYPERSURFACES OF $\mathbb{R}^{N+1}$
}

\author{
JAUME LLIBRE \\ Departament de Matemàtiques \\ Universitat Autònoma de Barcelona, 08193, Bellaterra, Barcelona, \\ Catalonia, Spain, e-mail: jllibre@mat.uab.es \\ YUDY BOLAÑOS \\ Departament de Matemàtiques, \\ Universitat Autònoma de Barcelona, 08193 Bellaterra, Barcelona, \\ Catalonia, Spain,e-mail: ymbolanos@gmail.com
}

July 20, 2011

Using sophisticated techniques of Algebraic Geometry Jouanolou in 1979 showed that if the number of invariant algebraic hypersurfaces of a polynomial vector field in $\mathbb{R}^{n}$ of degree $m$ is at least $\left(\begin{array}{c}n+m-1 \\ n\end{array}\right)+n$, then the vector field has a rational first integral. Llibre and Zhang used only Linear Algebra provided a shorter and easier proof of the result given by Jouanolou. We use ideas of Llibre and Zhang to extend the Jouanolou result to polynomial vector fields defined on algebraic regular hypersurfaces of $\mathbb{R}^{n+1}$, this extended result completes the standard results of the Darboux theory of integrability for polynomial vector fields on regular algebraic hypersurfaces of $\mathbb{R}^{n+1}$.

\section{Introduction}

In many branches of applied sciences appear nonlinear ordinary differential equations. For a differential system or a vector field defined in $\mathbb{R}^{2}$ the existence of a first integral determines completely its phase portrait. In $\mathbb{R}^{n}$ with $n>2$ the existence of a first integral of a vector field reduces the study of its dynamics in one dimension, with the time real or complex, respectively. So the following natural question arises: Given a vector field on $\mathbb{R}^{n}$, how to recognize if this vector field has a first integral? This question has no satisfactory answers up to now. Many different methods have been used for studying the existence of first integrals of vector fields. Some of these methods based on: Noether symmetries [Cantrijn \& Sarlet,1981], the Darboux theory of integrability [Darboux, 1878], the Lie symmetries [Olver, 1986], the Painlevé analysis [Bountis et al.,1984], the use of Lax pairs [Lax, 1968], the direct method [Giacomini et al.,1991, Hietarinta, 1987] and, the linear compatibility analysis method [Strelcyn \& Wojciechowski, 1988], the Carleman embedding procedure [Andrade \& Rauth, 1981, Carleman,1932], the quasimonomial formalism [Bountis et al.,1984], etc. For polynomial vector fields and rational first integrals the best answer to this question 
was given by Jouanolou [Jouanolou, 1979] in 1979 inside the Darboux theory of integrability. This theory of integrability provides a link between the integrability of polynomial vector fields and the number of invariant algebraic hypersurfaces that they have.

The objective of this paper is to extended the result of Jouanolou on the existence of rational first integrals of polynomial vector fields of $\mathbb{R}^{n+1}$ to polynomial vector fields defined on regular algebraic hypersurfaces of $\mathbb{R}^{n+1}$.

Darboux [Darboux, 1878] in 1878 showed how can be constructed a first integral of polynomial vector fields in $\mathbb{R}^{n}$ possessing sufficient invariant algebraic hypersurfaces. In particular he proved that if a polynomial vector field in $\mathbb{R}^{n}$ of degree $m$ has at least $\left(\begin{array}{c}m+n-1 \\ n\end{array}\right)+1$ invariant algebraic hypersurfaces, then it has a first integral, which can be computed using these invariant algebraic hypersurfaces. Jouanolou [Jouanolou, 1979] in 1979 showed, using sophisticated techniques of Algebraic Geometry, that if the number of invariant algebraic hypersurfaces of a polynomial vector field in $\mathbb{R}^{n}$ of degree $m$ is at least $\left(\begin{array}{c}n+m-1 \\ n\end{array}\right)+n$, then the vector field has a rational first integral, which also can be computed using the invariant algebraic hypersurfaces.

In [Llibre \& Zhang, 2010] Llibre and Zhang used only Linear Algebra for provided a shorter and easier proof of the result given by Jouanolou .

In this paper we use ideas of [Llibre \& Zhang, 2010] to extend the Jouanolou result to polynomial vector fields defined on algebraic regular hypersurfaces of $\mathbb{R}^{n+1}$.

En [Llibre \& Zhang, 2002] Llibre and Zhang extended the Darboux theory of integrability in $\mathbb{R}^{n}$ to polynomial vector fields on regular algebraic hypersurfaces of $\mathbb{R}^{n+1}$. However, the conditions for the existence of a rational first integral only are provided for the particular case of vector fields defined on the quadric surfaces and two-dimensional torus embedded in $\mathbb{R}^{3}$. So our extension of the Jouanolou's result completes the standard results of the Darboux theory of integrability for polynomial vector fields on regular algebraic hypersurfaces of $\mathbb{R}^{n+1}$.

The paper is organized as follows. In section 2 we provide some definitions and we state our main result. In section 3 we state some auxiliary results. Finally in section 4 we prove the extension of the
Jouanolou's result to polynomial vector fields on regular algebraic hypersurfaces of $\mathbb{R}^{n+1}$.

In fact all the results of this paper can be extended to polynomial vector fields in $\mathbb{C}^{n+1}$ but working with meromorphic first integrals, here we prefer to present the results in $\mathbb{R}^{n+1}$.

\section{Basic Concepts and Statement of the Main Result}

Let $\Omega$ be a hypersurface given by

$\Omega=\left\{\left(x_{1}, \ldots, x_{n+1}\right) \in \mathbb{R}^{n+1}: G\left(x_{1}, \ldots, x_{n+1}\right)=0\right\}$, where $G: \mathbb{R}^{n+1} \rightarrow \mathbb{R}$ is a $C^{1}$ map. Without loss of generality, by the Implicit Function Theorem we can assume that $x_{n+1}=g\left(x_{1}, \ldots, x_{n}\right)$ for all $\left(x_{1}, \ldots, x_{n+1}\right) \in \Omega$ except perhaps in a set of Lebesgue measure zero, with $g: \pi(\Omega) \rightarrow \mathbb{R}$ of class $C^{1}$ being $\pi: \mathbb{R}^{n+1} \rightarrow \mathbb{R}^{n}$ the projection along the $x_{n+1}$-axis, this is $\pi\left(x_{1}, \ldots, x_{n+1}\right)=\left(x_{1}, \ldots, x_{n}\right)$.

The hypersurface $\Omega$ is regular if the gradient of $G$ does not vanish on $\Omega$. We say that $\Omega$ is an algebraic hypersurface if $G$ is a polynomial. If the degree of the polynomial $G$ is $d$, then we say that $\Omega$ is algebraic of degree $d$. In the rest of this article all the hypersurfaces that appear are algebraic regular hypersurfaces.

Let $\mathcal{X}=\left(P_{1}, \ldots, P_{n+1}\right)$ be a polynomial vector field of $\mathbb{R}^{n+1}$ given by

$$
\mathcal{X}=\sum_{i=1}^{n+1} P_{i}\left(x_{1}, \ldots, x_{n+1}\right) \frac{\partial}{\partial x_{i}},
$$

where $\left(x_{1}, \ldots, x_{n+1}\right) \in \mathbb{R}^{n+1}$ and each $P_{i}$ for $i=$ $1, \ldots, n+1$ is a polynomial of degree at most $m$. We say that $\mathcal{X}$ defines a polynomial vector field on the regular hypersurface $\Omega$ if it satisfies that

$$
\mathcal{X} G=\left(P_{1}, \ldots, P_{n+1}\right) \cdot \nabla G=0
$$

on all points of $\Omega$.

The vector field $\mathcal{X}$ on $\Omega$, except for a set of Lebesgue measure zero, is also written making use of $x_{n+1}=g\left(x_{1}, \ldots, x_{n}\right)$, as $\tilde{\mathcal{X}}=\left(\tilde{P}_{1}, \ldots, \tilde{P}_{n}\right)$ satisfying $\tilde{\mathcal{X}} \tilde{G}=0$. In general, the notation $\tilde{f}$ applied to a function $f: A \subset \mathbb{R}^{n+1} \rightarrow \mathbb{R}$ represents the function $f$ restricted to $\Omega$, i.e.

$$
\begin{aligned}
\tilde{f} & =\tilde{f}\left(x_{1}, \ldots, x_{n}\right) \\
& =f\left(x_{1}, \ldots, x_{n}, g\left(x_{1}, \ldots, x_{n}\right)\right),
\end{aligned}
$$


where $\left(x_{1}, \ldots, x_{n}\right) \in \pi(A)$.

As usual we denote by $\mathbb{C}\left[x_{1}, \ldots, x_{n+1}\right]$ the ring of all polynomials in the variables $x_{1}, \ldots, x_{n+1}$ with coefficients in $\mathbb{C}$. Let $f\left(x_{1}, \ldots, x_{n+1}\right) \in$ $\mathbb{C}\left[x_{1}, \ldots, x_{n+1}\right] \backslash \mathbb{C}$. We say that $\{f=0\} \cap \Omega \subset \mathbb{R}^{n+1}$ is an algebraic invariant hypersurface of the vector field $\mathcal{X}$ on $\Omega$ (or simply an invariant algebraic hypersurface of $\Omega$ ) if it satisfies:

(i) There exists a polynomial $k \in \mathbb{C}\left[x_{1}, \ldots, x_{n+1}\right]$ such that

$$
\mathcal{X} f=\sum_{i=1}^{n+1} P_{i} \frac{\partial f}{\partial x_{i}}=k f \quad \text { on } \Omega,
$$

the polynomial $k$ is the cofactor of $f=0$ on $\Omega$;

(ii) the two hypersurfaces $f=0$ and $\Omega$ have transversal intersection; i.e. $\nabla G \wedge \nabla f \neq 0$ on the hypersurface $\{f=0\} \cap \Omega$, where $\wedge$ denotes the wedge product of two vectors in $\mathbb{R}^{n+1}$.

We remark that in the definition of algebraic invariant hypersurface $f=0$ we allow that this hypersurface be complex; that is $f \in \mathbb{C}\left[x_{1}, \ldots, x_{n+1}\right] \backslash \mathbb{C}$. This is due to the fact that sometimes for real polynomial vector fields the existence of a real first integral can be forced by the existence of complex invariant algebraic hypersurfaces. Thus sometimes the real integrability can occur by the underlying complex structure. If the polynomial vector field $\mathcal{X}$ is real and has a complex invariant algebraic hypersurface, then we know that its conjugate must also be an invariant algebraic hypersurface. Since the first integral that provides the Darboux theory uses conjugate pairs of these functions, the first integral obtained is real.

Let $f$ and $g$ polynomials of $\mathbb{C}_{m-1}\left[x_{1}, \ldots, x_{n+1}\right]$, i.e. the ring of all polynomials of $\mathbb{C}\left[x_{1}, \ldots, x_{n+1}\right]$ up to degree $m-1$. We say that $f$ and $g$ are related, $f \sim g$, if they coincide on $\Omega$, i.e. there is a polynomial $h$ of degree at most $m-d-1$ such that $f-g=h G$. This relationship defines the quotient space $\mathbb{C}_{m-1}\left[x_{1}, \ldots, x_{n+1}\right] / \sim$, we denote its dimension by $N=N(n, m, d)$, called the dimension of $\mathbb{C}_{m-1}\left[x_{1}, \ldots, x_{n+1}\right]$ on $\Omega$. The following result gives us this dimension, for a proof see for instance [Llibre \& Zhang, 2002].
Proposition 2.1. The dimension $N(n, m, d)$ of $\mathbb{C}_{m-1}\left[x_{1}, \ldots, x_{n+1}\right]$ on $\Omega$ is given by

$$
N(n, m, d)=\left(\begin{array}{c}
n+m \\
n+1
\end{array}\right)-\left(\begin{array}{c}
n+m-d \\
n+1
\end{array}\right) .
$$

We say that an invariant algebraic hypersurface $f=0$ is irreducible in $\mathbb{C}\left[x_{1}, \ldots, x_{n+1}\right]$ if the polynomial $f$ is irreducible in $\mathbb{C}\left[x_{1}, \ldots, x_{n+1}\right]$.

The next theorem is the main result of this article, it provides the conditions for determining if a polynomial vector field defined on a regular algebraic hypersurface $\Omega$ admits or not a rational first integral.

Theorem 2.2. Let $\Omega$ be a regular algebraic hypersurface of degree $d$ in $\mathbb{R}^{n+1}$. The polynomial vector field $\mathcal{X}$ on $\Omega$ of degree $m>0$ admits $N(n, m, d)+n$ invariant algebraic hypersurfaces irreducible in $\mathbb{C}\left[x_{1}, \ldots, x_{n+1}\right]$ if and only if $\mathcal{X}$ has a rational first integral on $\Omega$.

Under the assumptions of Theorem 2.2 all the orbits of the vector field $\mathcal{X}$ are contained in invariant algebraic hypersurfaces.

\section{Preliminary Results}

Before presenting the proof of main result we need to prove an auxiliary result. First we provide some concepts such as first integral and $k$-functionally independent first integrals for polynomial vector fields on regular algebraic hypersurfaces which we will need in the development of this theory.

Let $B$ be an open subset of $\mathbb{R}^{n+1}$ (or respectively $\Omega$ ). We denote by $\mu_{B}$ the Lebesgue measure of $\mathbb{R}^{n+1}$ (or respectively $\Omega$ ) restricted to $B$, thus $\mu_{B}(A)$ is the Lebesgue measure of the set $A \subset B$. We say that the Lebesgue measure of $A$ is full in $B$, or simply full if $\mu_{B}(B \backslash A)$ is zero.

Let $H_{i}: \mathbb{R}^{n+1} \rightarrow \mathbb{R}$ with $i=1, \ldots, p$ be functions of class $C^{1}$ defined in $\mathbb{R}^{n+1}$ except perhaps in a set of Lebesgue measure zero. We say that $H_{1}, \ldots, H_{p}$ are $k$-functionally independent in $\Omega$ if the rank of the matrix $n \times p$ formed by the gradients $\nabla \tilde{H}_{1}(x), \ldots, \nabla \tilde{H}_{p}(x)$ is $k$ for all $x=$ $\left(x_{1}, \ldots, x_{n+1}\right) \in \pi(\Omega)$ except perhaps in a set of Lebesgue measure zero in $\pi(\Omega)$. If $H_{1}, \ldots, H_{p}$ are $p$-functionally independent in $\Omega$ simply we say that they are functionally independent in $\Omega$. Of course, 
we say that $H_{1}, \ldots, H_{p}$ are functionally dependent on $\Omega$ if they are not functionally independent in $\Omega$.

Let $D \subset \Omega$ be an open subset of full Lebesgue measure in $\Omega$. We say that the function $H: D \rightarrow \mathbb{R}$ of class $C^{1}$ is a first integral of the polynomial vector field $\mathcal{X}$ on $\Omega$ if $\mathcal{X} H=0$ on $D$, or also $\tilde{\mathcal{X}} \tilde{H}=0$ on $\pi(D)$. If moreover $H$ is a rational function in variables $x_{1}, \ldots, x_{n+1}$ of $\mathbb{R}^{n+1}$, then $H$ is called a rational first integral of the vector field $\mathcal{X}$ on $\Omega$.

Theorem 3.1. Let $H_{1}, \ldots, H_{p}$ be first integrals of class $C^{2}$ of the polynomial vector field $\mathcal{X}$ of $\mathbb{R}^{n+1}$ on the hypersurface $\Omega \subset \mathbb{R}^{n+1}$ given by (1). Assume that $H_{1}, \ldots, H_{p}$ defined on a subset $D$ of $\Omega$ of full measure in $\Omega$ are $k$-functionally independent on $\Omega$. Without loss of generality we can assume that $\nabla \tilde{H}_{1}, \ldots, \nabla \tilde{H}_{k}$ are functionally independent.

(a) For each $s \in\{k+1, \ldots, p\}$ there exist functions $C_{s 1}(x), \ldots, C_{s k}(x)$ of class $C^{1}$ defined on a full Lebesgue measurable subset of $\Omega$ such that

$\nabla H_{s}(x)=C_{s 1}(x) \nabla H_{1}(x)+\cdots+C_{s k}(x) \nabla H_{k}(x)$.

(b) For every $s \in\{k+1, \ldots, p\}$ and $j \in\{1, \ldots, k\}$ the function $C_{s j}(x)$ (if not a constant) is a first integral of $\mathcal{X}$ on $\Omega$.

Proof. (a) By the hypotheses the gradients $\nabla \tilde{H}_{1}(x), \ldots, \nabla \tilde{H}_{k}(x)$ are linearly independent for all $x \in \pi(D)$, and for each $s \in\{k+$ $1, \ldots, p\}$ the vector $\nabla \tilde{H}_{s}(x)$ is linearly dependent of $\nabla \tilde{H}_{1}(x), \ldots, \nabla \tilde{H}_{k}(x)$ for all $x$ in a set $\tilde{D}$ of full Lebesgue measure in $\pi(D)$. Therefore there are functions $\left\{\tilde{C}_{s 1}(x), \ldots, \tilde{C}_{s k}(x)\right\}$ defined in $\tilde{D}$ such that

$$
\nabla \tilde{H}_{s}(x)=\tilde{C}_{s 1}(x) \nabla \tilde{H}_{1}(x)+\cdots+\tilde{C}_{s k}(x) \nabla \tilde{H}_{k}(x) .
$$

From which we get the system of size $n \times k$

$$
\begin{gathered}
\left(\begin{array}{ccc}
\frac{\partial \tilde{H}_{1}(x)}{\partial x_{1}} & \cdots & \frac{\partial \tilde{H}_{k}(x)}{\partial x_{1}} \\
\vdots & \vdots & \vdots \\
\frac{\partial \tilde{H}_{1}(x)}{\partial x_{n}} & \cdots & \frac{\partial \tilde{H}_{k}(x)}{\partial x_{n}}
\end{array}\right)\left(\begin{array}{c}
\tilde{C}_{s 1}(x) \\
\vdots \\
\tilde{C}_{s k}(x)
\end{array}\right) \\
=\left(\begin{array}{c}
\frac{\partial \tilde{H}_{s}(x)}{\partial x_{1}} \\
\vdots \\
\frac{\partial \tilde{H}_{s}(x)}{\partial x_{n}}
\end{array}\right) .
\end{gathered}
$$

Since the dimension of $\Omega$ is $n$, the maximum number of independent first integrals of the vector field on $\Omega$ is at most $n-1$, this is $k \leq n-1$. Thus from the system (3) we can extract a subsystem whose associated matrix $A_{s}(x)$ of size $k \times k$ has nonzero determinant. Assume that this matrix $A_{s}(x)$ consists of the rows $i_{1}, \ldots, i_{k}$ of the matrix of $(3)$. We can apply the Cramer rule and find the solutions of the subsystem with matrix $A_{s}(x)$, i.e.

$$
\tilde{C}_{s i}(x)=\frac{\operatorname{det} A_{s}^{i}(x)}{\operatorname{det} A_{s}(x)}
$$

where $A_{s}^{i}(x)$ is the matrix obtained by replacing the $i$-th column of $A_{s}(x)$ for the column vector $\left(\frac{\partial \tilde{H}_{s}(x)}{\partial x_{i_{1}}}, \ldots, \frac{\partial \tilde{H}_{s}(x)}{\partial x_{i_{k}}}\right)^{T}$.

Since the functions $\tilde{H}_{j}$ with $j=1, \ldots, p$ are first integrals of class $C^{2}$ and they are defined on the set $\tilde{D}$ of full Lebesgue measure in $\Omega$, the functions $\tilde{C}_{s 1}(x), \ldots, \tilde{C}_{s k}(x)$ defined over $\tilde{D}$ are of class $C^{1}$, this completes the proof of statement $(a)$.

(b) Let $x \in \tilde{D}$. For all $i, j \in\{1, \ldots, n\}$, from (3) we have

$$
\frac{\partial \tilde{H}_{s}}{\partial x_{i}}=\tilde{C}_{s 1} \frac{\partial \tilde{H}_{1}}{\partial x_{i}}+\cdots+\tilde{C}_{s k}(x) \frac{\partial \tilde{H}_{k}}{\partial x_{i}}
$$

and

$$
\frac{\partial \tilde{H}_{s}}{\partial x_{j}}=\tilde{C}_{s 1} \frac{\partial \tilde{H}_{1}}{\partial x_{j}}+\cdots+\tilde{C}_{s k}(x) \frac{\partial \tilde{H}_{k}}{\partial x_{j}} .
$$

We derive these two equations with respect to $x_{j}$ and $x_{i}$ respectively and we obtain

$$
\begin{aligned}
\frac{\partial}{\partial x_{j}} \frac{\partial \tilde{H}_{s}}{\partial x_{i}}= & \frac{\partial \tilde{C}_{s 1}}{\partial x_{j}} \frac{\partial \tilde{H}_{1}}{\partial x_{i}}+\tilde{C}_{s 1} \frac{\partial^{2} \tilde{H}_{1}}{\partial x_{j} \partial x_{i}}+\cdots \\
& +\frac{\partial \tilde{C}_{s k}}{\partial x_{j}} \frac{\partial \tilde{H}_{k}}{\partial x_{i}}+\tilde{C}_{s k} \frac{\partial^{2} \tilde{H}_{k}}{\partial x_{j} \partial x_{i}} \\
\frac{\partial}{\partial x_{i}} \frac{\partial \tilde{H}_{s}}{\partial x_{j}}= & \frac{\partial \tilde{C}_{s 1}}{\partial x_{i}} \frac{\partial \tilde{H}_{1}}{\partial x_{j}}+\tilde{C}_{s 1} \frac{\partial^{2} \tilde{H}_{1}}{\partial x_{i} \partial x_{j}}+\cdots \\
& +\frac{\partial \tilde{C}_{s k}}{\partial x_{i}} \frac{\partial \tilde{H}_{k}}{\partial x_{j}}+\tilde{C}_{s k} \frac{\partial^{2} H_{k}}{\partial x_{i} \partial x_{j}} .
\end{aligned}
$$

Subtracting these two equations we get

$$
\begin{aligned}
& \frac{\partial \tilde{C}_{s 1}}{\partial x_{i}} \frac{\partial \tilde{H}_{1}}{\partial x_{j}}-\frac{\partial \tilde{C}_{s 1}}{\partial x_{j}} \frac{\partial \tilde{H}_{1}}{\partial x_{i}}+\cdots \\
& +\frac{\partial \tilde{C}_{s k}}{\partial x_{i}} \frac{\partial \tilde{H}_{k}}{\partial x_{j}}-\frac{\partial \tilde{C}_{s k}}{\partial x_{j}} \frac{\partial \tilde{H}_{k}}{\partial x_{i}}=0 .
\end{aligned}
$$


Since $k \leq n-1$, we consider two cases. First, we suppose that $k=n-1$. From (4) we obtain

$$
\begin{array}{r}
\sum_{1 \leq i<j \leq n}\left(\left(\frac{\partial \tilde{C}_{s 1}}{\partial x_{i}} \frac{\partial \tilde{H}_{1}}{\partial x_{j}}-\frac{\partial \tilde{C}_{s 1}}{\partial x_{j}} \frac{\partial \tilde{H}_{1}}{\partial x_{i}}+\cdots\right.\right. \\
\left.+\frac{\partial \tilde{C}_{s k}}{\partial x_{i}} \frac{\partial \tilde{H}_{k}}{\partial x_{j}}-\frac{\partial \tilde{C}_{s k}}{\partial x_{j}} \frac{\partial \tilde{H}_{k}}{\partial x_{i}}\right) \\
\sum_{\sigma\left(k_{1}, k_{2}, \ldots, k_{n-2}\right)}(-1)^{\tau\left(i j k_{1}, k_{2} \ldots k_{n-2}\right)} \frac{\partial \tilde{H}_{2}}{\partial x_{k_{1}}} \frac{\partial \tilde{H}_{3}}{\partial x_{k_{2}}} \\
\left.\cdots \frac{\partial \tilde{H}_{n-1}}{\partial x_{k_{n-2}}}\right)=0
\end{array}
$$

where $\sigma$ is a permutation of $\{1, \ldots, n\} \backslash\{i, j\}$. We take the second term over all possible permutations and $\tau$ evaluated on a permutation of $\{1, \ldots, n\}$ is the minimum number of transpositions for passing the permutation to the identity.

We can write the equality (5) as

$$
\begin{aligned}
& \sum_{1 \leq i<j \leq n}\left(\frac{\partial \tilde{C}_{s 1}}{\partial x_{i}} \frac{\partial \tilde{H}_{1}}{\partial x_{j}}-\frac{\tilde{C}_{s 1}}{\partial x_{j}} \frac{\partial \tilde{H}_{1}}{\partial x_{i}}\right) \cdot \\
& \sum_{\sigma\left(k_{1}, \ldots, k_{n-2}\right)}(-1)^{\tau\left(i j k_{1} \ldots k_{n-2}\right)} \frac{\partial \tilde{H}_{2}}{\partial x_{k_{1}}} \cdots \frac{\partial \tilde{H}_{n-1}}{\partial x_{k_{n-2}}} \\
& \sum_{1 \leq i<j \leq n}\left(\frac{\partial \tilde{C}_{s k}}{\partial x_{i}} \frac{\partial \tilde{H}_{k}}{\partial x_{j}}-\frac{\partial \tilde{C}_{s k}}{\partial x_{j}} \frac{\partial \tilde{H}_{k}}{\partial x_{i}}\right) \cdot \\
& \sum_{\sigma\left(k_{1} \ldots, k_{n-2}\right)}(-1)^{\tau\left(i j k_{1} \ldots k_{n-2}\right)} \frac{\partial \tilde{H}_{2}}{\partial x_{k_{1}}} \cdots \frac{\partial \tilde{H}_{n-1}}{\partial x_{k_{n-2}}} \\
& =0 .
\end{aligned}
$$

Using Laplace's rule for the calculation of a determinant we have

$$
\begin{aligned}
\sum_{1 \leq i<j \leq n}\left(\frac{\partial \tilde{C}_{s 1}}{\partial x_{i}} \frac{\partial \tilde{H}_{1}}{\partial x_{j}}-\frac{\tilde{C}_{s 1}}{\partial x_{j}} \frac{\partial \tilde{H}_{1}}{\partial x_{i}}\right) \cdot \\
=\left|\begin{array}{cccc}
\frac{\partial \tilde{C}_{s 1}}{\partial x_{1}} & \frac{\partial \tilde{C}_{s 1}}{\partial x_{2}} & \cdots & \frac{\partial \tilde{C}_{s 1}}{\partial x_{n}} \\
\frac{\partial \tilde{H}_{1}}{\partial x_{1}} & \frac{\partial \tilde{H}_{1}}{\partial x_{2}} & \cdots & \frac{\partial \tilde{H}_{1}}{\partial x_{n}} \\
\frac{\partial \tilde{H}_{2}}{\partial x_{1}} & \frac{\partial \tilde{H}_{2}}{\partial x_{2}} & \cdots & \frac{\partial \tilde{H}_{2}}{\partial x_{k_{n-2}}} \\
\vdots & \vdots & \ddots & \vdots \\
\frac{\partial \tilde{H}_{n-1}}{\partial x_{1}} & \frac{\partial \tilde{H}_{n-1}}{\partial x_{2}} & \cdots & \frac{\partial \tilde{H}_{n-1}}{\partial x_{n}}
\end{array}\right|
\end{aligned}
$$

and for $l=2, \ldots, k$ we obtain

$$
\begin{aligned}
& \sum_{1 \leq i<j \leq n}\left(\frac{\partial \tilde{C}_{s l}}{\partial x_{i}} \frac{\partial \tilde{H}_{l}}{\partial x_{j}}-\frac{\partial \tilde{C}_{s l}}{\partial x_{j}} \frac{\partial \tilde{H}_{l}}{\partial x_{i}}\right) \\
& \sum_{\sigma\left(k_{1} \ldots, k_{n-2}\right)}(-1)^{\tau\left(i j k_{1} \ldots k_{n-2}\right)} \frac{\partial \tilde{H}_{2}}{\partial x_{k_{1}}} \cdots \frac{\partial \tilde{H}_{n-1}}{\partial x_{k_{n-2}}}
\end{aligned}
$$

$$
=\left|\begin{array}{cccc}
\frac{\partial \tilde{C}_{s l}}{\partial x_{1}} & \frac{\partial \tilde{C}_{s l}}{\partial x_{2}} & \ldots & \frac{\partial \tilde{C}_{s l}}{\partial x_{n}} \\
\frac{\partial \tilde{H}_{l}}{\partial x_{1}} & \frac{\partial \tilde{H}_{l}}{\partial x_{2}} & \ldots & \frac{\partial \tilde{H}_{l}}{\partial x_{n}} \\
\frac{\partial \tilde{H}_{2}}{\partial x_{1}} & \frac{\partial \tilde{H}_{2}}{\partial x_{2}} & \cdots & \frac{\partial \tilde{H}_{2}}{\partial x_{n}} \\
\vdots & \vdots & \ddots & \vdots \\
\frac{\partial \tilde{H}_{n-1}}{\partial x_{1}} & \frac{\partial \tilde{H}_{n-1}}{\partial x_{2}} & \cdots & \frac{\partial \tilde{H}_{n-1}}{\partial x_{n}}
\end{array}\right|=0 .
$$

Therefore equation (5) can be written as

$$
\left|\begin{array}{cccc}
\frac{\partial \tilde{C}_{s 1}}{\partial x_{1}} & \frac{\partial \tilde{C}_{s 1}}{\partial x_{2}} & \cdots & \frac{\partial \tilde{C}_{s 1}}{\partial x_{n}} \\
\frac{\partial \tilde{H}_{1}}{\partial x_{1}} & \frac{\partial \tilde{H}_{1}}{\partial x_{2}} & \cdots & \frac{\partial \tilde{H}_{1}}{\partial x_{n}} \\
\vdots & \vdots & \ddots & \vdots \\
\frac{\partial \tilde{H}_{n-1}}{\partial x_{1}} & \frac{\partial \tilde{H}_{n-1}}{\partial x_{2}} & \cdots & \frac{\partial \tilde{H}_{n-1}}{\partial x_{n}}
\end{array}\right|=0 .
$$

From (6) we have that for every $x \in \tilde{D}$, the vector $\nabla \tilde{C}_{s 1}$ belongs to the vector space $(n-1)$ dimensional generated by $\nabla \tilde{H}_{1}(x), \ldots, \nabla \tilde{H}_{n-1}(x)$ denoted by $\mathcal{P}_{n-1}(x)$, because $k=n-1$ and $\nabla \tilde{H}_{1}(x), \ldots, \nabla \tilde{H}_{k}(x)$ are linearly independent.

By definition of first integral in $\Omega$, we have for each $x \in \tilde{D}$,

$$
\frac{\partial \tilde{H}_{j}(x)}{\partial x_{1}} \tilde{P}_{1}(x)+\cdots+\frac{\partial \tilde{H}_{j}(x)}{\partial x_{n}} \tilde{P}_{n}(x)=0,
$$

with $j=1, \ldots, n-1$.

So for every $x \in \tilde{D}$, the vector $\tilde{\mathcal{X}}(x)$ is orthogonal to the space $\mathcal{P}_{n-1}(x)$, and hence $\nabla \tilde{C}_{s 1}$ is orthogonal to $\tilde{\mathcal{X}}(x)$, i.e.

$$
\frac{\partial \tilde{C}_{s 1}}{\partial x_{1}} \tilde{P}_{1}(x)+\cdots+\frac{\partial \tilde{C}_{s 1}(x)}{\partial x_{n}} \tilde{P}_{n}(x)=0,
$$

for all $x \in \tilde{D}$.

This proves that the function $\tilde{C}_{s 1}$ (if not a constant) is a first integral of the vector field $\tilde{\mathcal{X}}$ defined on $\tilde{D}$, that is, $C_{s 1}$ is a first integral of $\mathcal{X}$ on $\Omega$. 
Similar arguments can be used to prove that the functions $\tilde{C}_{s j}$ (if not a constant) are also first integrals of $\mathcal{X}$ on $\Omega$ for $j=2, \ldots, k$. Therefore the statement $(b)$ is proved for $k=n-1$.

Now we assume $k<n-1$. Taking into account that $H_{1}, \ldots, H_{k}$ are functionally independent in $D$ and working in a similar way to the case $k=n-1$ we have

$$
\operatorname{rank}\left(\begin{array}{cccc}
\frac{\partial \tilde{C}_{s 1}}{\partial x_{1}} & \frac{\partial \tilde{C}_{s 1}}{\partial x_{2}} & \cdots & \frac{\partial \tilde{C}_{s 1}}{\partial x_{n}} \\
\frac{\partial \tilde{H}_{1}}{\partial x_{1}} & \frac{\partial \tilde{H}_{1}}{\partial x_{2}} & \cdots & \frac{\partial \tilde{H}_{1}}{\partial x_{n}} \\
\vdots & \vdots & \vdots & \vdots \\
\frac{\partial \tilde{H}_{k}}{\partial x_{1}} & \frac{\partial \tilde{H}_{k}}{\partial x_{2}} & \cdots & \frac{\partial \tilde{H}_{k}}{\partial x_{n}}
\end{array}\right)=k
$$

instead of (6). This means that for all $x \in \tilde{D}, \nabla \tilde{C}_{s 1}$ belongs to the vector space $k$-dimensional generated by $\left\{\nabla \tilde{H}_{1}(x), \ldots, \nabla \tilde{H}_{k}(x)\right\}$, denoted by $\mathcal{P}_{k}(x)$.

Since the functions $\tilde{H}_{j}(x)$ for $j=1, \ldots, k$ are first integrals of the vector field $\tilde{\mathcal{X}}$, the vector $\tilde{\mathcal{X}}(x)$ is orthogonal to $\nabla \tilde{C}_{s 1}(x)$ for all $x \in D$. So the function $\tilde{C}_{s 1}$ (if not a constant) is a first integral of the field $\mathcal{X}$ on $\Omega$.

Using similar arguments we can show that the functions $\tilde{C}_{s j}$ for $j=2, \ldots, k$, are also first integrals of $\mathcal{X}$ on $\Omega$. This completes the proof of statement (b) of the theorem.

\section{Proof of Main Result}

In this section we shall prove our main result.

Proof of Theorem 2.2. First, we suppose that the polynomial vector field $\mathcal{X}$ has a rational first integral $H$ on $\Omega$ given by

$$
H\left(x_{1}, \ldots, x_{n+1}\right)=\frac{g_{1}\left(x_{1}, \ldots, x_{n+1}\right)}{g_{2}\left(x_{1}, \ldots, x_{n+1}\right)},
$$

with $\left(x_{1}, \ldots, x_{n+1}\right) \in \Omega$ and $g_{1}, g_{2} \in$ $\mathbb{R}\left[x_{1}, \ldots, x_{n+1}\right]$. Let $h_{1}=H\left(y_{1}, \ldots, y_{n+1}\right)$ for some $\left(y_{1}, \ldots, y_{n+1}\right) \in \Omega$. We define

$$
\begin{aligned}
& f\left(x_{1}, \ldots, x_{n+1}\right)= \\
& \quad g_{1}\left(x_{1}, \ldots, x_{n+1}\right)-h_{1} g_{2}\left(x_{1}, \ldots, x_{n+1}\right) .
\end{aligned}
$$

Note that all points $\left(x_{1}, \ldots, x_{n+1}\right)$ contained in the hypersurface $f=0$ satisfy that $H\left(x_{1}, \ldots, x_{n+1}\right)=$ $h_{1}$. Then, since $H$ is a first integral of $\mathcal{X}$ on $\Omega$, if a trajectory of $\mathcal{X}$ on $\Omega$ has a point on $f=0$, the full trajectory is contained in $f=0$. That is, we obtain that $f=0$ is an invariant algebraic hypersurface $\mathcal{X}$ on $\Omega$.

Since for every $\left(y_{1}, \ldots, y_{n+1}\right) \in \Omega$ we can define an invariant algebraic hypersurface of the vector field $\mathcal{X}$ on $\Omega$, we obtain the proof of the "if" part of the theorem.

Now we prove the "only if" part. We write $N=$ $N(n, m, d)$. Let $f_{i}(x)=0$ for $i=1, \ldots, N+n$ be invariant algebraic hypersurfaces of the vector field $\mathcal{X}$ on $\Omega$ with cofactor $k_{i}(x)$. So $\mathcal{X} f_{i}(x)=k_{i}(x) f_{i}(x)$ on $\Omega$, i.e. $\left.\mathcal{X} f_{i}(x)\right|_{\Omega}=\left.\left.k_{i}(x)\right|_{\Omega} f_{i}(x)\right|_{\Omega}$. Therefore we can assume that $k_{i}(x) \in \mathbb{C}_{m-1}\left[x_{1}, \ldots, x_{n+1}\right] / \sim$. Let $q$ be the dimension of the vector subspace of $\mathbb{C}_{m-1}\left[x_{1}, \ldots, x_{n+1}\right] / \sim$ generated by $\left\{k_{1}(x), \ldots, k_{N+n}(x)\right\}$. Then, by Proposition 2.1 we have $q \leq N$. In order to simplify the proof and the notation we will assume that $q=N$ and that $k_{1}(x), \ldots, k_{N}(x)$ are linearly independent in $\mathbb{C}_{m-1}\left[x_{1}, \ldots, x_{n+1}\right] / \sim$. If $q<N$ the proof would be similar using the same arguments.

For every $j \in\{1, \ldots, n\}$ there exists a vector $\left(\sigma_{j 1}, \ldots, \sigma_{j N}, 1\right) \in \mathbb{C}^{N+1}$ such that

$$
\sigma_{j 1} k_{1}(x)+\ldots+\sigma_{j N} k_{N}(x)+k_{N+j}(x)=0,
$$

on $\Omega$. From the definition of the invariant algebraic hypersurface $f_{j}=0$ we get that $k_{j}=\mathcal{X} f_{j} / f_{j}$. Then from (7) we obtain

$$
\sigma_{j 1} \frac{\mathcal{X} f_{1}(x)}{f_{1}(x)}+\ldots+\sigma_{j N} \frac{\mathcal{X} f_{N}(x)}{f_{N}}+\frac{\mathcal{X} f_{N+j}(x)}{f_{N+j}(x)}=0 .
$$

As $\mathcal{X}\left(f_{j}^{\sigma_{j}}\right)=\sigma_{j} f_{j}^{\sigma_{j}-1} \mathcal{X}\left(f_{j}\right)$ we have $\frac{\mathcal{X}\left(f_{j}^{\sigma_{j}}\right)}{f_{j}^{\sigma_{j}}}=$ $\sigma_{j} \frac{\mathcal{X}\left(f_{j}\right)}{f_{j}}$. Then

$$
\frac{\mathcal{X}\left(f_{1}^{\sigma_{j 1}}\right)}{f_{1}^{\sigma_{j 1}}}+\ldots+\frac{\mathcal{X}\left(f_{N}^{\sigma_{j N}}\right)}{f_{N}^{\sigma_{j N}}}+\frac{\mathcal{X}\left(f_{N+j}\right)}{f_{N+j}}=0
$$

thus

$\mathcal{X}\left(\log f_{1}^{\sigma_{j 1}}\right)+\ldots+\mathcal{X}\left(\log f_{N}^{\sigma_{j N}}\right)+\mathcal{X}\left(\log f_{N+j}\right)=0$,

and hence

$$
\mathcal{X}\left(\log \left(f_{1}^{\sigma_{j 1}} \ldots f_{N}^{\sigma_{j N}} f_{N+j}\right)\right)=0,
$$

for $j=1, \ldots, n$ on $\Omega$. This means that for $j=$ $1, \ldots, n$ the functions $H_{j}=\log \left(f_{1}^{\sigma_{j 1}} \ldots f_{N}^{\sigma_{j N}} f_{N+j}\right)$ 
are first integrals of the vector field $\mathcal{X}$ on $\Omega$, defined on a convenient full Lebesgue measurable subset $D_{1} \subset \Omega$.

We claim that the $n$ first integrals $H_{j}$ 's are functionally dependent on any subset of $\Omega$ with positive Lebesgue measure. Now we prove the claim. Assume that there exists a Lebesgue measurable subset $D_{2} \subset \Omega$ with positive measure in $\Omega$ such that the functions $H_{j}$ 's are functionally independent in $D_{2}$. Then from the definition of first integral we have

$$
\frac{\partial \tilde{H}_{j}(x)}{\partial x_{1}} \tilde{P}_{1}(x)+\cdots+\frac{\partial \tilde{H}_{j}(x)}{\partial x_{n}} \tilde{P}_{n}(x)=0,
$$

for $j=1, \ldots, n$ and for every $x \in \pi\left(D_{2}\right)$. And from the definition functional independence this homogeneous linear system of size $n \times n$ has the trivial solution $\tilde{P}_{j}(x)=0$ for $j=1, \ldots, n$ on $\pi\left(D_{2}\right)$, and consequently the vector field $\mathcal{X}=0$ in $\Omega$. This contradicts the assumption that $\mathcal{X}$ has degree $m>0$. So the claim is proved.

We define $r(x)=\operatorname{rank}\left\{\nabla \tilde{H}_{1}(x), \ldots, \nabla \tilde{H}_{n}(x)\right\}$ and $k=\max \left\{r(x): x \in \pi\left(D_{1}\right)\right\}$. Then there exists an open subset $\mathcal{O}$ of $\pi\left(D_{1}\right)$ such that $k=r(x)$ for all $x \in \mathcal{O}$ and $k<n$. Thus, the $n$ first integrals $H_{j}$ 's are $k$-functionally independent on $\Omega$. Without loss of generality we can assume that

$$
\operatorname{rank}\left(\begin{array}{cccc}
\frac{\partial \tilde{H}_{1}}{\partial x_{1}} & \frac{\partial \tilde{H}_{1}}{\partial x_{2}} & \cdots & \frac{\partial \tilde{H}_{1}}{\partial x_{n}} \\
\vdots & \vdots & \vdots & \vdots \\
\frac{\partial \tilde{H}_{k}}{\partial x_{1}} & \frac{\partial \tilde{H}_{k}}{\partial x_{2}} & \cdots & \frac{\partial \tilde{H}_{k}}{\partial x_{n}}
\end{array}\right)=k,
$$

for all $x \in \mathcal{O}$.

By Theorem 3.1( $a$ ) for every $x \in \mathcal{O} \subset D_{1}$ there exist $C_{s 1}(x), \ldots, C_{s N}(x)$ such that

$$
\nabla H_{s}(x)=C_{s 1}(x) \nabla H_{1}(x)+\cdots+C_{s k}(x) \nabla H_{k}(x)
$$

for each $s=k+1, \ldots, n$. And by Theorem 3.1(b) we have that the function $C_{s j}(x)$ (if not a constant) for $j \in\{1, \ldots, k\}$ is a first integral of the vector field $\mathcal{X}$ on $\mathcal{O} \subset \Omega$.

Since $H_{i}=\log \left(f_{1}^{\sigma_{i 1}} \ldots f_{N}^{\sigma_{i N}} f_{N+i}\right)$ we know that each $\nabla H_{i}$ is a vector of rational functions. Moreover the vectors $\nabla H_{1}(x), \ldots, \nabla H_{k}(x)$ are linearly independent for each $x \in \mathcal{O}$, then solving system (8) we get a unique solution $\left(C_{s 1}, \ldots, C_{s k}\right)$ on $\mathcal{O}$ for each $s=k+1, \ldots, n$. So each function $C_{s j}$ for $j=1, \ldots, k$ and $s=k+1, \ldots, n$ is rational. Since $\mathcal{O}$ is an open subset of $\Omega$ and $C_{s j}$ is rational, it should satisfy the last equation in $\Omega$ except possibly a subset of Lebesgue measure zero where $C_{s j}$ is not defined. Hence if some of the functions $C_{s j}(x)$ 's is not a constant, it is a rational first integral of the vector field $\mathcal{X}$ on $\Omega$.

Now we will prove that some function $C_{s j}$ is not a constant. Equation (8) implies that if all the functions $C_{s 1}, \ldots, C_{s k}$ are constants then

$$
H_{s}(x)=C_{s 1} H_{1}(x)+\cdots+C_{s k} H_{k}(x)+\log C_{s},
$$

for $s=k+1, \ldots, n$ and $C_{s}$ is a constant. So

$$
\begin{aligned}
\log \left(f_{1}^{\sigma_{s 1}} \ldots f_{N}^{\sigma_{s N}} f_{N+s}\right)= & \\
& C_{s 1} \log \left(f_{1}^{\sigma_{11}} \ldots f_{N}^{\sigma_{1 N}} f_{N+1}\right)+\cdots+ \\
& C_{s k} \log \left(f_{1}^{\sigma_{k 1}} \ldots f_{N}^{\sigma_{k N}} f_{N+k}\right)+\log C_{s} .
\end{aligned}
$$

From this last equation we obtain

$$
\begin{aligned}
f_{1}^{\sigma_{s 1}} \ldots f_{N}^{\sigma_{s N}} f_{N+s}= & \left(f_{1}^{\sigma_{11}} \ldots f_{N}^{\sigma_{1 N}} f_{N+1}\right)^{C_{s 1}} \ldots \\
& \left(f_{1}^{\sigma_{k 1}} \ldots f_{N}^{\sigma_{k N}} f_{N+k}\right)^{C_{s k}} C_{s}
\end{aligned}
$$

and therefore

$$
\begin{aligned}
f_{N+s}= & C_{s} f_{1}^{C_{s 1} \sigma_{11}+\cdots+C_{s k} \sigma_{k 1}-\sigma_{s 1}} \cdots \\
& f_{N}^{C_{s 1} \sigma_{1 N}+\cdots+C_{s k} \sigma_{k 1}-\sigma_{s N}} f_{N+1}^{C_{s 1}} \cdots f_{N+k}^{\sigma_{s k}}
\end{aligned}
$$

for all $s \in\{k+1, \ldots, n\}$. This contradicts the fact that the polynomials $f_{1}, \ldots, f_{N+n}$ are irreducible and pairwise different. Hence we must have a nonconstant function $C_{s_{0} j_{0}}(x)$ for some $j_{0} \in\{1, \ldots, k\}$ and some $s_{0} \in\{k+1, \ldots, n\}$. This completes the proof of Theorem 2.2.

\section{When $\Omega$ is a Quadric or a 2-Torus}

As mentioned in the introduction, in [Llibre \& Zhang, 2002] were presented the extension of the Darboux theory of integrability to polynomials vector fields defined on a quadric or on a bidimensional torus. It is well known that the quadrics can be classified into seventeen types. Since here we only consider real polynomial vector fields defined on real hypersurfaces, we omit the five types of imaginary quadrics. Also we omit the three types of quadrics that are formed by planes since the study of the Darboux theory of integrability of the vector fields on these planes is reduced to the classic study of the Darboux 


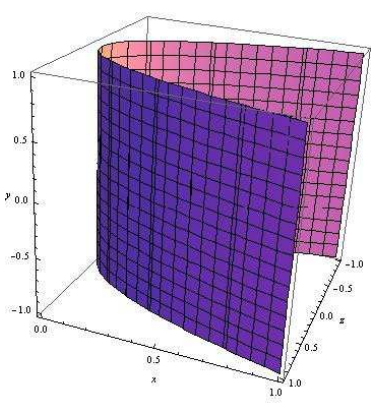

Parabolic cylinder: $z^{2}-x=0$

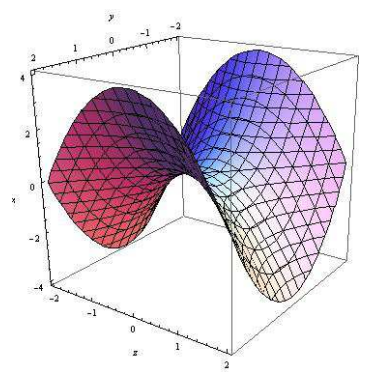

Hyperbolic paraboloid: $y^{2}-z^{2}-x=0$

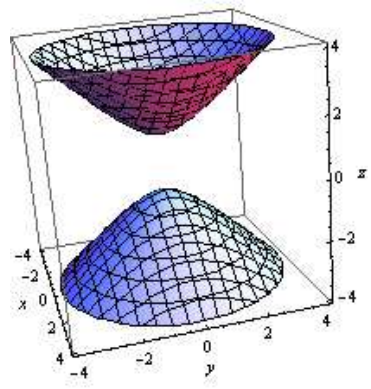

Hyperboloid of two sheets:

$x^{2}+y^{2}-z^{2}+1=0$

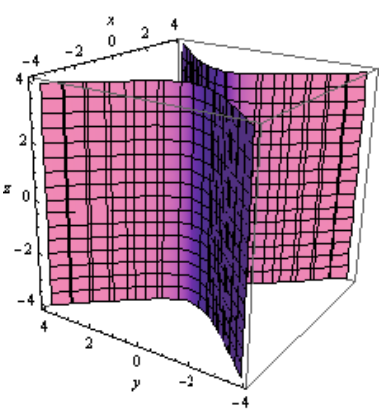

Hyperbolic cylinder: $x^{2}-z^{2}-1=0$

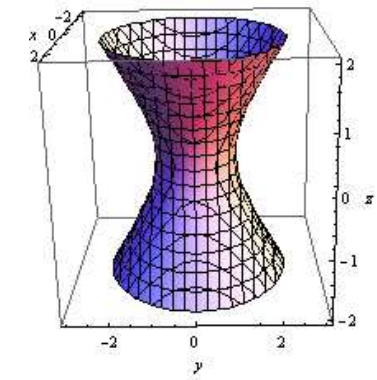

Hyperboloid of one sheet:

$$
x^{2}+y^{2}-z^{2}-1=0
$$

Fig. 1. Quadrics and their canonical forms.

theory of integrability of planar polynomial vector fields. Thus we only work with the remainder nine quadrics. The conditions that guarantee the existence of rational first integrals of a polynomial vector field defined over one of these nine quadrics and the 2-dimensional torus were given in [Llibre \& Zhang, 2002]. Here we reobtain these conditions as an application of Theorem 2.2. In Figures 1 and 2 are represented the nine quadrics considered here, the bidimensional torus and their canonical forms. Now we calculate the number of

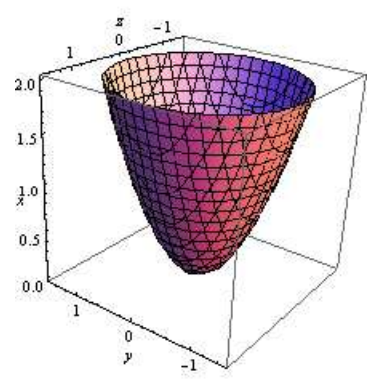

Elliptic paraboloid:

$$
y^{2}+z^{2}-x=0
$$

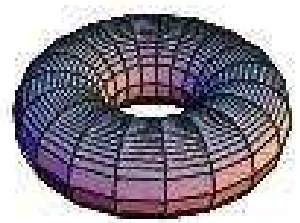

2-dimensional torus: $z^{2}+\left(\sqrt{x^{2}+y^{2}}-a\right)^{2}=1$ $a>1$.

Fig. 2. Quadrics, torus and their canonical forms.

invariant algebraic curves necessary for guarantee the existence of a rational first integral of a polynomial vector field $\mathcal{X}$ of degree $m$ in $\mathbb{R}^{3}$ when it is defined on a quadric $(d=2)$ or a bidimensional torus $(d=4)$.

By Proposición 2.1 we have that

$$
N(n, m, d)=\left(\begin{array}{c}
n+m \\
n+1
\end{array}\right)-\left(\begin{array}{c}
n+m-d \\
n+1
\end{array}\right) .
$$

For the quadrics $n+1=3, d=2$ and $m \geq 3$, so

$$
N(2, m, 2)=\left(\begin{array}{c}
2+m \\
3
\end{array}\right)-\left(\begin{array}{c}
m \\
3
\end{array}\right)=m^{2},
$$

in the case of 2 -torus we have that $n+1=3, d=4$ and $m \geq 5$, thus

$$
N(2, m, 4)=\left(\begin{array}{c}
2+m \\
3
\end{array}\right)-\left(\begin{array}{c}
m-2 \\
3
\end{array}\right)=2 m^{2}-4 m+4 .
$$

So from Theorem 2.2 results that $N(2, m, 2)+2=$ $m^{2}+2$ is the number of invariants algebraic curves that guarantee the existence of a rational first integral for the case of a polynomial vector field of 
degree $m \geq 3$ defined on a quadric. If the polynomial vector field of degree $m \geq 5$ is defined on a bidimensional torus the minimum number of invariant algebraic curves is $N(2, m, 4)+2=2 m^{2}-$ $4 m+6$. These results agree with those obtained in [Llibre \& Zhang, 2002].

\section{Some Algebraic Surfaces $\Omega$}

Now we shall apply Theorem 2.2 to polynomial vector fields of $\mathbb{R}^{3}$ defined on well known algebraic surfaces. For each surface we determine the number of invariant algebraic curves which guarantee the existence of a rational first integral.

\subsection{Fermat cubic}

Assume that $\Omega=\{G=0\}$ is the Fermat cubic surface [Ness, 1978], i.e. $x^{3}+y^{3}+z^{3}=1$. It is easy to see that this surface is regular since $\nabla G$ vanishes only at the origin but this point is not in the surface. The surface is represented in Figure 3. Applying Proposición 2.1 we calculate $N(n, m, d)$.

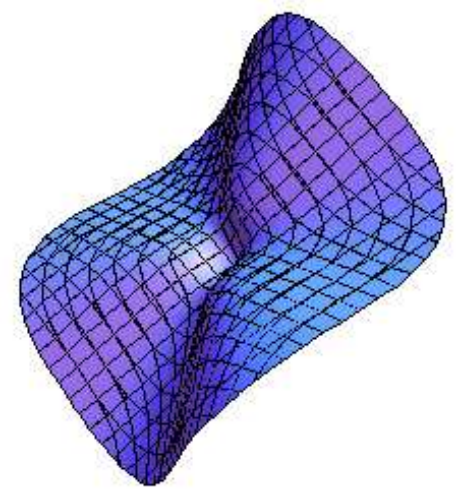

Fig. 3. Fermat cubic

In this case we have that $n=2, m \geq 4$ and $d=3$. Thus,

$$
\begin{aligned}
N(2, m, 3) & =\left(\begin{array}{c}
m+2 \\
3
\end{array}\right)-\left(\begin{array}{c}
m-1 \\
3
\end{array}\right) \\
& =\frac{1}{2}\left(3 m^{2}-3 m+2\right) .
\end{aligned}
$$

Then from (9) and by Theorem 2.2 we can conclude that the number of invariant algebraic curves necessary for a polynomial vector field $\mathcal{X}$ of degree $m \geq 4$ defined on the Fermat cubic surface possesses a rational first integral is $N(2, m, 3)+2=$ $3\left(m^{2}-m+2\right) / 2$.

\subsection{Clebsch surface}

Let $\Omega=\{G=0\}$ the surface of Clebsch [Hirzebruch, 1976] given by

$$
x^{3}+y^{3}+z^{3}+1-(x+y+z+1)^{3}=0 .
$$

Its gradient is

$$
\begin{aligned}
\nabla G= & x^{2}-3(1+x+y+z)^{2}, 3 y^{2}- \\
& \left.3(1+x+y+z)^{2}, 3 z^{2}-3(1+x+y+z)^{2}\right),
\end{aligned}
$$

which vanishes at the points

$$
\begin{aligned}
& \left(\frac{-1}{2}, \frac{-1}{2}, \frac{ \pm 1}{2}\right),\left(\frac{-1}{2}, \frac{1}{2}, \frac{-1}{2}\right), \\
& \left(\frac{-1}{4}, \frac{-1}{4}, \frac{-1}{4}\right),\left(\frac{1}{2}, \frac{-1}{2}, \frac{-1}{2}\right) .
\end{aligned}
$$

Since none of these points are on the surface we obtain that $G$ is regular. This surface is represented in Figure 4. In this case we have that $n=2$,

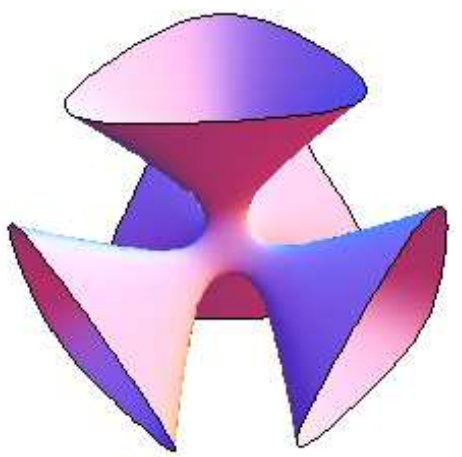

Fig. 4. Clebsh surface

$m \geq 4$ and $d=3$. Thus $N(n, m, d)$ corresponds to the same value obtained for the Fermat surface (9). Then by Theorem 2.2 we can conclude that the number of invariant algebraic curves necessary for a polynomial vector field $\mathcal{X}$ of degree $m \geq 4$ defined on the Clebsh surface possesses a first integral rational is $N(2, m, 3)+2=3\left(m^{2}-m+2\right) / 2$. 


\subsection{Goursat surface}

Let $\Omega=\{G=0\}$ the Goursat surface [Goursat, 1897] given by

$$
x^{4}+y^{4}+z^{4}-\left(x^{2}+y^{2}+z^{2}\right)=1 .
$$

Its gradient is the vector

$$
\nabla G=\left(-2 x+4 x^{3},-2 y+4 y^{3},-2 z+4 z^{3}\right),
$$

which vanishes in 27 points that do not belong to the surface. Therefore $\Omega$ is a regular surface. This surface is represented in Figure 5. Here we have

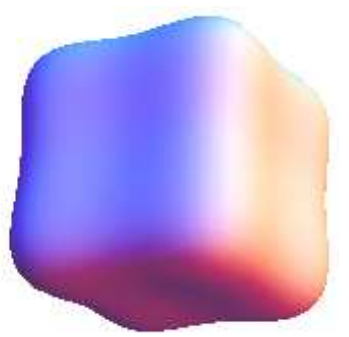

Fig. 5. Goursat surface

that $n=2, m \geq 5$ and $d=4$, thus $N(n, m, d)$ corresponds to the value obtained for the case of the bidimensional torus, i.e. $N(2, m, 4)=2 m^{2}-4 m+4$.

Then, by Theorem 2.2, the number of invariant algebraic curves required for a polynomial vector field $\mathcal{X}$ of degree $m \geq 5$ defined on the Goursat surface $\Omega$ has a rational first integral is $N(2, m, 4)+$ $2=2 m^{2}-4 m+6$.

\subsection{Superficie Racor}

Let us show that the surface $G(x, y, z)=x^{2} y^{2}+$ $x^{2} z^{2}+y^{2} z^{2}-1$ is regular in $\mathbb{R}^{3}$. Its gradient is given by $\nabla G=\left(2 x\left(y^{2}+z^{2}\right) 2 y\left(x^{2}+z^{2}\right), 2 z\left(x^{2}+y^{2}\right)\right)$. Since $\nabla G$ does not vanish at any point of the surface we obtain that $G$ is regular. This surface is represented in Figure 6.

In this case we have that $n=2, m \geq 5$ and $d=$ 4. Thus, the number of invariant algebraic curves required for a polynomial vector field $\mathcal{X}$ of degree $m \geq 5$ defined on the algebraic surface $G$ possesses a rational first integral is the same as obtained for the Goursat surface.

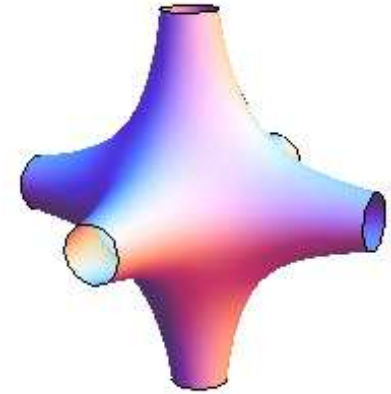

Fig. 6. Racor surface

\section{Acknowledgements}

The first author is supported by the grants MICIIN/FEDER MTM 2008-03437, AGAUR grant 2009SGR410, and is partially supported by ICREA Academia.

\section{References}

Andrade, R.F.S., \& Rauth, A., [1981] "The Lorenz model and the method of Carleman embedding," Phys. Lett. A 82, 276-278.

Bountis, T.C.,Ramani A., Grammaticos, B. \& Dorizzi, B., [1984] "On the complete and partial integrability of non-Hamiltonian systems," J. Phys. A 128, 268-288.

Carleman, T. [1932] "Application de la théoie des équations intégrales linéaires aux systémes d'équations différentielles non linéaires," Acta Mathematica 59, 63-87.

Cantrijn, F., \& Sarlet, W., [1981] "Generalizations of Noether's theorem in classical mechanics," SIAM Rev. 23, 467-494.

Darboux, G., [1878] "De l'emploi des solutions particulières algébriques dans l'intégration des systèmes d'équations différentielles algébriques," C. R. Math. Acad. Sci. París 86, 533-536, 584589, 1012-1014.

Giacomini, H.J., Repetto, C.E., \& Zandron, O.P., [1991] "Integrals of motion of three-dimensional non Hamiltonian dynamical systems," J. Phys. A 24, 4567-4574. 
Hietarinta, J., [1987] "Direct methods for the search of the second invariant," Phys. Rep. 147, 87154.

Jouanolou, J.P., [1979] Equations de Plaff algébriques Lecture Notes in Mathematics 708 Springer, New York.

Goursat, E., [1897] "Étude des surfaces qui admettent tous les plans de symétrie d'un polyèdre régulier," Ann. Sci. École Norm. Sup. 4, 1592000.

Hirzebruch, F., [1976] "The Hilbert modular group for the field $\mathbb{Q}(\sqrt{5})$ and the cubic diagonal surface of Clebsch and Klein," Russian Math. Surveys 31 (5), 96-110.

Lax, P.D., [1968] "Integrals of nonlinear equations of evolution and solitary waves," Commun. Pure Appl. Math. 21, 467-490..

Llibre, J., \& Rodríguez, G., [2002] "Darboux integrability of polynomial vector fields on 2dimensional surfaces," Internat. J. Bifur. Chaos Appl. Sci. Engrg. 12, 2821-2833.

Llibre, J., \& Zhang, X., [2002] "Darboux integrability of real polynomial vector fields on regular algebraic hypersurfaces," Rendiconti del Circolo Matematico di Palermo 51, 109-126.

Llibre, J., \& Zhang, X., [2010] "Rational first integrals in the Darboux theory of integrability in $\mathbb{C}^{n}, "$ Bulletin des Sciences mathématiques 134, 189-195.

Ness, L., [1978] "Curvature on the Fermat cubic," Duke Mathematical Journal 45 (4), 797-807.

Olver,P.J., [1986] Applications of Lie Groups to Differential Equations Springer, New York.

Strelcyn, J.M., \& Wojciechowski, S., [1988] "A method of finding integrals for threedimensional dynamical systems," Phys. Lett. A 133, 207-212. 